This item was submitted to Loughborough's Research Repository by the author.

Items in Figshare are protected by copyright, with all rights reserved, unless otherwise indicated.

\title{
Calculation of ground vibration spectra from heavy military vehicles
}

PLEASE CITE THE PUBLISHED VERSION

http://dx.doi.org/10.1016/j.jsv.2010.02.014

PUBLISHER

(C) Elsevier

VERSION

AM (Accepted Manuscript)

LICENCE

CC BY-NC-ND 4.0

REPOSITORY RECORD

Krylov, Victor V., S. Pickup, and J. McNuff. 2012. "Calculation of Ground Vibration Spectra from Heavy Military Vehicles". figshare. https://hdl.handle.net/2134/10788. 
This item was submitted to Loughborough's Institutional Repository (https://dspace.lboro.ac.uk/) by the author and is made available under the following Creative Commons Licence conditions.

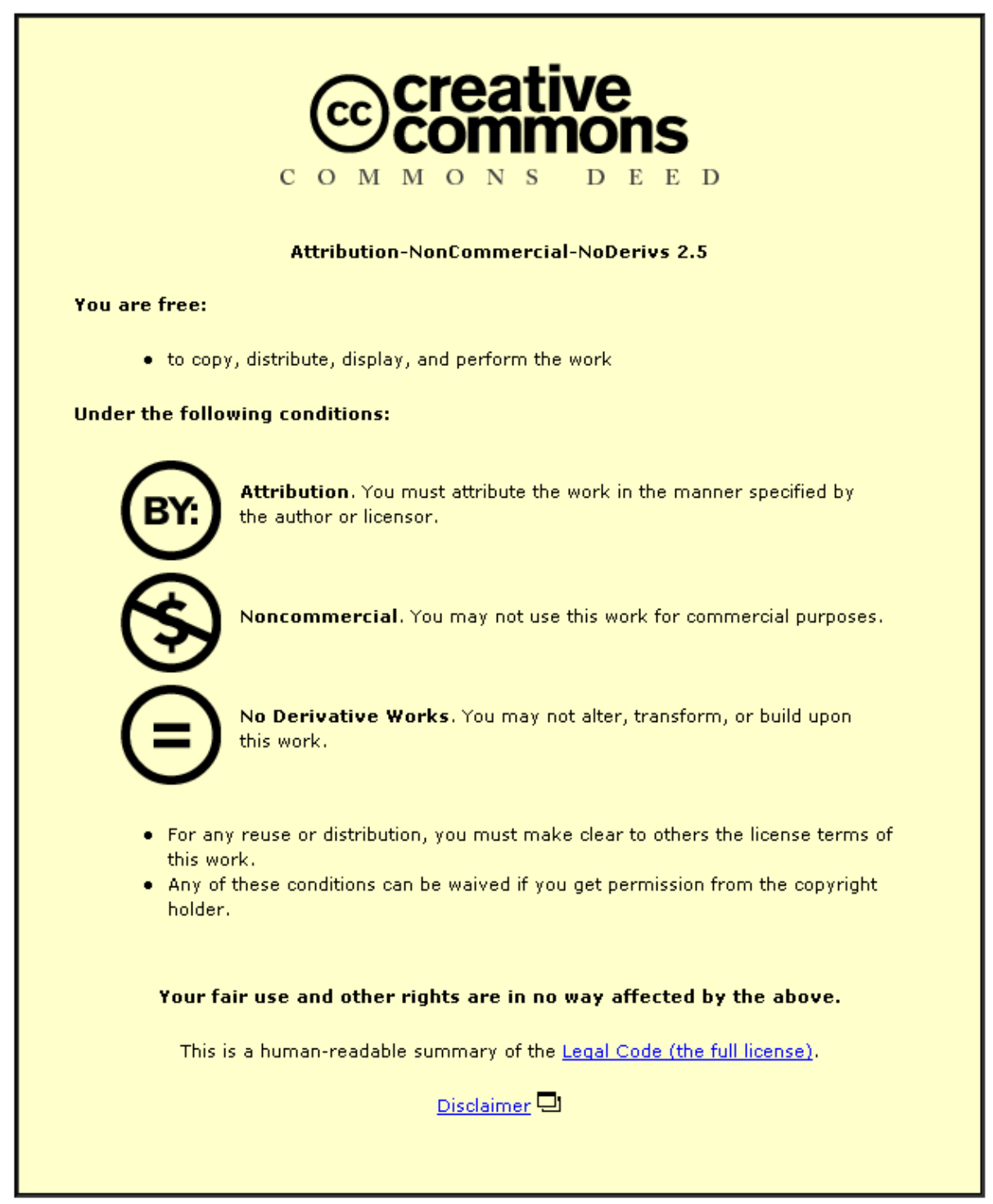

For the full text of this licence, please go to: http://creativecommons.org/licenses/by-nc-nd/2.5/ 


\title{
Calculation of ground vibration spectra from heavy
}

\section{military vehicles}

\author{
V.V. Krylov* , S. Pickup, J. McNuff \\ Department of Aeronautical and Automotive Engineering, \\ Loughborough University, \\ Loughborough, Leicestershire LE11 3TU, UK
}

\section{Abstract}

The demand for reliable autonomous systems capable to detect and identify heavy military vehicles becomes an important issue for UN peacekeeping forces in the current delicate political climate. A promising method of detection and identification is the one using the information extracted from ground vibration spectra generated by heavy military vehicles, often termed as their seismic signatures. This paper presents the results of the theoretical investigation of ground vibration spectra generated by heavy military vehicles,

\footnotetext{
* Corresponding author. Tel.: +44 1509 227216;

E-mail address: V.V.Krylov@lboro.ac.uk
} 
such as tanks and armed personnel carriers. A simple quarter car model is considered to identify the resulting dynamic forces applied from a vehicle to the ground. Then the obtained analytical expressions for vehicle dynamic forces are used for calculations of generated ground vibrations, predominantly Rayleigh surface waves, using Green's function method. A comparison of the obtained theoretical results with published experimental data shows that analytical techniques based on the simplified quarter car vehicle model are capable of producing ground vibration spectra of heavy military vehicles that reproduce basic properties of experimental spectra.

Keywords: Ground vibrations, Seismic signatures, Heavy military vehicles, Quarter car model, Green’s function method.

\section{Introduction}

Ground vibrations are traditionally studied in the fields of civil engineering and environmental acoustics [1, 2]. More recently though, they have been investigated also for the purposes of remote detection and monitoring of heavy military vehicles [3-6]. In particular, the roles of generated ground vibration spectra (also termed as seismic signatures) have been studied experimentally in the framework of the so-called Bochum Verification Project (BVP) [3, 4]. This project was one of the first academic investigations into this topic that was open for participation of scientists from several European countries, USA and Russia. Whilst acoustic monitoring of vehicles, which was investigated in the BVP as well, would allow detection at much greater distances than those typical for seismic 
methods, seismic monitoring can provide additional possibilities to identify specific vehicle parameters and hence the types of vehicles.

It is envisaged that a typical application of the technology would be for UN peace keeping forces - to monitor agreed limits concerning cease-fire lines and weapons free zones, disarmament treaties, etc. Currently it is typical for only major routes to be staffed by inspectors, with other areas regulated through spot-checks and patrols. This leaves vast off-road portions of land that provide ample opportunity for prohibited movements. Autonomous acoustic and seismic sensors would provide covert monitoring, be independent of time-of-day or weather, and maximise coverage. A well-orchestrated network of sensors could provide gap-free monitoring, detecting suspicious activity and alerting a common control centre. The typical distance between sensors in the network is defined by typical Rayleigh wave propagation distance at these frequencies, which is around $100 \mathrm{~m}$ (see [3, 4]). This form of monitoring would prove less intrusive than a permanent human presence, and providing the systems are cost-effective, would demonstrate substantial financial benefit.

This paper aims to explore some fundamental characteristics of ground vibration spectra that could be attributed to heavy military vehicles traversing over flat terrain. Unlike works of other researchers, who employed either experimental methods $[3,4]$ or purely numerical approaches $[5,6]$, the present paper adopts mainly analytical techniques in order to describe the dynamic motion of typical heavy vehicles and to determine the forces applied from vehicles to the ground surface. These forces are then used for derivation of analytical expressions for generated ground vibrations, predominantly Rayleigh surface waves, using Green's function method. The advantage of such an analytical approach is that in case of a simple homogeneous soil structure it assists in better understanding of basic properties of seismic signatures of heavy military vehicles and its dependence on different parameters. 
For more complex soil layering though and in particular in case of ground being inhomogeneous in horizontal direction, numerical methods remain indispensable.

A simplified quarter car model (QCM) representation of a heavy vehicle is considered. For this representation, the dynamic forces applied from a vehicle to the ground are derived in the vehicle 'body still' approximation and then used for calculation of generated ground vibrations. The model simulates the effect of tyre or track geometrical irregularities (discontinuities) on generating ground vibrations. It is shown that the obtained ground vibration spectra contain spectral peaks associated with vehicle characteristic parameters and vehicle speed. A comparison of the obtained theoretical results with published experimental data shows that the above-mentioned analytical techniques based on the simplified quarter car vehicle model and Green’s function method are capable of producing ground vibration spectra of heavy military vehicles that reproduce basic properties of experimental spectra.

\section{Calculation of vehicle-induced ground forces}

Ground vibrations generated as a result of heavy vehicle motion over terrain can be attributed to the dynamic forces applied directly to the ground and to the conversion of vehicle-generated air borne sound into the ground motion via acousto-seismic coupling.

The dynamic forces applied directly to the ground are:

- Forces exerted to the ground as a result of wheel motion over ground disturbances or track (tyre) periodic irregularities [4, 7].

- Unbalanced forces due to engine and drive rotation that are transmitted to the vehicle body and then to the ground. 
- Forces exerted to the ground as a result of vehicle acceleration or braking $[7,8]$.

The conversion of vehicle-generated air borne sound into the ground motion via acousto-seismic coupling takes place when sound waves travel along the ground. This effect is stronger with porous soil. In this case, the varying air pressure produces a slow acoustic wave travelling nearly vertically into the soil pores. Friction then sets the soil matrix into motion $[4,9]$.

In the present paper, only the first type of directly applied forces is considered, namely the forces exerted to the ground as a result of wheel motion over track or tyre periodic irregularities.

\subsection{Ground force spectra for a simplified quarter car vehicle model}

A simple quarter car model (QCM) has been used to simulate the contact forces exerted to the ground as a result of a vehicle motion over surfaces characterised by the presence of geometrical irregularities (see Fig. 1). For tracked vehicles moving over perfectly flat ground, these irregularities are due to the small gaps between track links. For wheeled vehicles, tyre treads can induce a similar effect. Several assumptions have been made to justify QCM as a valid vehicle simplification [7]:

1. A point contact patch assumption is deemed sufficient as typical wavelengths of generated Rayleigh waves are greater than the characteristic dimensions of a vehicle.

2. Total vehicle mass is distributed evenly over all the wheel stations at all times.

3. The road surface is considered rigid for the purpose of finding ground forces, as are the track links for tracked vehicle models. 
For the QCM shown in Fig. 1, the magnitude of the force $F_{t}$ applied to the ground is equivalent to the force exerted by the compression of the tyre spring due to the vertical displacement of the wheel. Therefore, the solution for the dynamic response of the wheel $\mathrm{z}_{\mathrm{w}}(\mathrm{t})$ to an input from the road irregularity $\mathrm{z}_{\mathrm{r}}(\mathrm{t})$ is required to determine such ground forces.

In the time domain, the input into the quarter car model under consideration is the elevation changes $\mathrm{z}_{\mathrm{r}}(\mathrm{t})$ as a result of the wheel's passing over track linkages and treads for tracked and wheeled vehicles respectively. To model the unevenness $\mathrm{z}_{\mathrm{r}}(\mathrm{t})$ experienced by a wheel passing over the vehicle's track is not an easy task. Strictly speaking, one has to consider a complex problem of wheel interaction (via a rubber tyre) with a tensioned chain of track links that in turn interacts with the real ground. In this paper, this complex problem is not considered. Instead, the simplest model of track-induced unevenness with non-sine periodicity has been chosen at this stage. For simplicity, the variation in surface profile over which a wheel (modelled as a point contact) traverses can be estimated as a finite series of half sine wave pulses with a frequency $f_{\text {tr }}$ corresponding to the ratio of the vehicle forward velocity $\mathrm{V}$ to the track pitch a: $\mathrm{f}_{\mathrm{tr}}=\mathrm{V} / \mathrm{a}$. By carrying out a simple Fourier integration over a certain time interval $\mathrm{T}$ (period of observation), this input signal can be represented in the frequency domain: $z_{r}(\omega)$. The corresponding expressions for $z_{r}(t)$ and $z_{r}(\omega)$ take the form

$$
\begin{aligned}
& z_{r}(t)= \begin{cases}z_{r_{\max }} \sin \left(2 \pi f_{t r} t\right) & \text { for } z_{r}(t) \geq 0 \\
0 & \text { otherwise }\end{cases} \\
& z_{r}(\omega)=\int_{-T / 2}^{T / 2} z_{r}(t) e^{-i \omega t} d t
\end{aligned}
$$


As a 2-DOF system, QCM responds well at both ‘wheel hop' and 'body bounce’ natural frequencies. To simplify QCM even more, one can consider the so-called 'body still' approximation [7] that reduces QCM to a 1-DOF system by freezing the low-frequency 'body bounce' mode of vibration (around 1-3 Hz). As a result, the problem is reduced to the analysis of only the wheel hop response to the displacement input from surface discontinuities that takes place at higher frequencies. This is usually sufficient for calculation of generated ground vibrations since they are generated more efficiently at higher frequencies [7] (see below for more detail).

Keeping this in mind, we use the simplified QCM, considering vehicle body as immobile in vertical direction and taking into account only axle vibrations. This 1-DOF simplified model comprises an unsprung (wheel) mass $\mathrm{M}_{\mathrm{w}}$ (this mass also includes masses of the suspension and of the shock absorber as well as a half of the axle mass) and two springs with constants $K_{t}$ and $K_{s}$ modelling respectively the rubber tyre compliance and the stiffness of vehicle suspension (Fig. 1).

According to the above-mentioned simplified QCM, the equation describing vertical displacements of each wheel $\mathrm{z}_{\mathrm{w}}$ versus its static position has the form

$$
M_{w} \frac{\partial^{2} z_{w}}{\partial t^{2}}+B_{s} \frac{\partial z_{w}}{\partial t}+K z_{w}=K_{t} z_{r}(t)
$$

where $\mathrm{K}=\mathrm{K}_{\mathrm{t}}+\mathrm{K}_{\mathrm{s}}$ is a combined elasticity of tyre and suspension, and $\mathrm{B}_{\mathrm{s}}$ is the damping coefficient of suspension (tyre damping is neglected). 
Assuming that the axle length is small as compared with wavelengths of generated ground vibrations and its centre is located at $\mathrm{x}=0$ and $\mathrm{y}=0$, the resulting normal concentrated force $F_{t}(t)$ applied from the axle to the ground can be written in the form

$$
F_{t}(t)=2 K_{t}\left[z_{w}(t)-z_{r}(t)\right]
$$

where the factor 2 takes into account that there are two wheels in an axle.

Solving Eqn (3) by Fourier method, one can obtain the following expression for the Fourier transform $\mathrm{z}_{\mathrm{w}}(\omega)$ of an axle vertical displacement $\mathrm{z}_{\mathrm{w}}(\mathrm{t})$ :

$$
Z_{w}(\omega)=z_{r}(\omega) Z(\omega)
$$

Here

$$
Z(\omega)=\frac{\omega_{t}^{2}}{\sqrt{\left(\omega_{0}^{2}-\omega^{2}\right)^{2}+(2 \omega \alpha)^{2}}} \exp \left[-i \tan ^{-1}\left(\frac{2 \omega \alpha}{\omega_{0}^{2}-\omega^{2}}\right)\right]
$$

is the frequency response function, where $\omega_{0}=\left(\mathrm{K} / \mathrm{M}_{\mathrm{w}}\right)^{1 / 2}$ is the wheel hop resonant frequency, $\omega_{t}=\left(K_{t} / M_{w}\right)^{1 / 2}$ is the tyre 'jumping' resonant frequency, $\alpha=B_{s} / 2 M_{w}$ is a normalised damping coefficient, and $\mathrm{z}_{\mathrm{r}}(\omega)$ is the Fourier spectrum corresponding to the irregularity profile. The Fourier transform of the vertical force applied from the vehicle to the ground, $F_{t}(\omega)$, is easily obtained from (4) via replacing $z_{w}(t)$ and $z_{r}(t)$ by their Fourier spectra and using Eqn (5):

$$
F_{t}(\omega)=2 K_{t}\left[z_{r}(\omega) Z(\omega)-z_{r}(\omega)\right]
$$


Note that for tracked vehicles the values of tyre compliance $K_{t}$, characterising the elasticity of solid rubber coating of road wheels, are quite high in comparison with the stiffness of suspension $K_{s}$. In this case the wheel follows the track irregularities $z_{r}$, and the dynamic forces applied to the ground through the track are defined mainly by the stiffness of suspension $K_{s}$. Indeed, as follows from Eqn (6), if to assume that $K_{t}>>K_{s}$, the transfer function $\mathrm{Z}(\omega)$ becomes

$$
Z(\omega) \approx \frac{K_{t}}{K_{t}+K_{s}} \approx 1
$$

and, according to Eqn (5), $\mathrm{z}_{\mathrm{w}} \approx \mathrm{z}_{\mathrm{r}}$.

Substituting Eqn (8) into Eqn (7), one obtains

$$
F_{t}(\omega) \approx 2 K_{t}\left[\frac{K_{t}}{K_{t}+K_{s}}-1\right] z_{r}(\omega)=-2 \frac{K_{t} K_{s}}{K_{t}+K_{s}} z_{r}(\omega) \approx-2 K_{s} z_{r}(\omega),
$$

as expected.

The QCM model described above is valid for modelling a single axle wheel displacement. To establish the ground force spectra observed due to the effects of multiple axles, a simple superposition of all wheel hop displacement responses should be taken. Obviously, the wheel hop response at each axle differs only by a phase shift that corresponds to the distance of the additional wheel axle (characterised by the integer number $\mathrm{n}$ ) from the front axle, $\mathrm{E}_{1 \mathrm{n}}$, divided by the vehicle forward speed $\mathrm{V}$ (see Reference [7]). The resulting expression for the vertical force $F_{z}{ }^{m w}(\omega)$ applied to the ground from the entire vehicle then takes the form 


$$
F_{Z}{ }^{m w}(\omega)=F_{t}(\omega) \cdot\left(1+\exp \left(i \omega \frac{E_{12}}{V}\right)+\exp \left(i \omega \frac{E_{13}}{V}\right) \ldots+\exp \left(i \omega \frac{E_{1 N}}{V}\right)\right)
$$

where $F_{t}(\omega)$ is the force spectrum for a single wheel axle defined by Eqn (7), and $N$ is the number of axles in a vehicle.

\subsection{Calculated ground force spectra for the test vehicle parameters}

Let us now introduce the two main 'test vehicles', on which most of the theoretical calculations of this paper are based. These are the Leopard-1 Main Battle Tank (MBT) and the Transportpanzer (Fuchs) Armoured Personnel Carrier (APC) - see Fig. 2. For the former, a set of experimental results for generated ground vibration velocity spectra is available as part of the published works following from the Bochum Verification Project (BVP) [4]. The parameters of the test vehicles that have been used in calculations of the present work are shown in Table 1.

Note that there is a lack of published information about the values of suspension stiffness and tyre compliance for the above-mentioned heavy military vehicles. The information that is available provides only some general characteristics, such as maximum speed, total vehicle mass, geometrical dimensions of tracks, etc. (see for example [10]). Therefore, the values of the above-mentioned two technical parameters in Table 1 have been obtained via theoretical estimates and by comparison with published parameters of similar vehicles (see e.g. $[5,6]$ ). The uncertainty in these parameters though is not 
detrimental for calculations of ground vibration spectra as it affects only amplitudes of the frequency peaks but not their positions.

Initially, the effects of single-axle and multi-axle inputs on the resulting ground force spectra have been analysed. Figures 3 and 4 illustrate the resulting single-axle and multiaxle ground force spectra respectively for the Leopard-1 MBT (with 7 axles) calculated using the simplified quarter car model (QCM). Frequency resolution used in calculations was $0.1 \mathrm{~Hz}$. As expected, in Figs 3 and 4 the most significant force peaks are at the main frequency of excitation $\mathrm{f}_{\mathrm{tr}}=\mathrm{V} / \mathrm{a}=23.1 \mathrm{~Hz}$ corresponding to the forward speed of the vehicle $\mathrm{V}=3.9 \mathrm{~m} / \mathrm{s}$ and the track pitch $\mathrm{a}=0.169 \mathrm{~m}$. Noticeable force amplitudes are observed also at integer multiples of this fundamental frequency (harmonics). Obviously, the amplitudes of all frequency peaks are proportional to the track hump height and depend on its shape.

The effect of multiple axles (see Fig. 4) produces the additional peaks at different harmonics of the so-called wheel-base frequency $\mathrm{f}_{\mathrm{wb}}=\mathrm{V} / \mathrm{E}_{12}=5.9 \mathrm{~Hz}$, where $\mathrm{E}_{12}=0.665$ $\mathrm{m}$ is the distance between two neighbouring axles.

Figure 5 shows the resulting multi-axle ground force spectrum calculated for the Transportpanzer (Fuchs) APC (with 3 unequally spaced axles). The obtained results show that amplitudes of the ground forces in this case are substantially smaller than for the tank, approximately $10 \%$ of the maximum tank force amplitude can be observed. The frequency of the main force peak in this case is around $156 \mathrm{~Hz}$ - this corresponds to the forward speed of the vehicle $\mathrm{V}=3.9 \mathrm{~m} / \mathrm{s}$ divided by the much smaller distance between the tyre tread elements $\mathrm{a}=0.025 \mathrm{~m}$.

\section{Calculation of generated ground vibrations}




\subsection{General comments}

To calculate ground vibration spectra generated by the vehicle-induced ground forces one can use Green's function method in which one can take into account only generated Rayleigh surface waves as they carry most of the radiated elastic energy (see Reference [7] for more detail). Under such circumstances, the vertical component of generated ground vibration velocity in the frequency domain, $v_{z}(\omega)$, can be described by the following expression [7]:

$$
v_{z}(\omega)=\left(\frac{2 \pi}{k_{R} \rho}\right)^{1 / 2} \frac{(-i \omega) k_{R} k_{t}^{2} v_{l}}{2 \pi \mu F^{\prime}\left(k_{R}\right)} F_{z}^{m w}(\omega) e^{-k_{R} \gamma \rho} e^{i k_{R} \rho-i 3 \pi / 4} .
$$

Here $\mathrm{F}_{\mathrm{z}}^{\mathrm{mw}}(\omega)$ is the multi-axle force spectrum defined by Eqn (10); $\rho=\rho(\mathrm{x}, \mathrm{y})$ is the distance to the observation point; $k_{R}=\omega / c_{R}$ is the Rayleigh wave number, where $c_{R}$ is Rayleigh wave velocity in the ground; $F^{\prime}\left(k_{R}\right)$ is the derivative $d F(k) / d k$ of the so-called Rayleigh determinant $\mathrm{F}(\mathrm{k})=\left(2 \mathrm{k}^{2}-\mathrm{k}_{\mathrm{t}}^{2}\right)^{2}-4 \mathrm{k}^{2} \mathrm{v}_{\mathrm{l}} \mathrm{v}_{\mathrm{t}}$ taken at $\mathrm{k}=\mathrm{k}_{\mathrm{R}}$, where $\mathrm{v}_{\mathrm{l}, \mathrm{t}}=\left(\mathrm{k}_{\mathrm{R}}^{2}\right.$ $\left.\mathrm{k}_{\mathrm{l}, \mathrm{t}}^{2}\right)^{1 / 2}$ are unspecified expressions; $\mathrm{k}_{\mathrm{l}, \mathrm{t}}=\omega / \mathrm{c}_{\mathrm{l}, \mathrm{t}}$ are the wavenumbers of bulk longitudinal and shear seismic waves, where $c_{l}$ and $c_{t}$ are their phase velocities.

In writing Eqn (11) we have taken account of attenuation of generated ground vibrations in the ground by replacing the wavenumber of a Rayleigh wave in an ideal elastic medium $k_{R}=\omega / c_{R}$ by the complex wavenumber $k_{R}{ }^{\prime}=k_{R}(1+i \gamma)=\left(\omega / c_{R}\right)(1+i \gamma)$. Here $\gamma<<1$ is a positive constant, called a loss factor, that describes the linear dependence of a Rayleigh wave attenuation coefficient on frequency $\omega$. For different types of ground $\gamma$ 
are in the range from 0.01 to 0.2 . In what follows we will be interested only in amplitudes of ground vibrations $\mathrm{V}_{\mathrm{z}}(\omega)=\left|\mathrm{v}_{\mathrm{z}}(\omega)\right|$, ignoring the phase information.

Keeping in mind that $F^{\prime}\left(k_{R}\right)$ is proportional to $\omega^{3}$, one can see that the whole expression in front of $\mathrm{F}_{\mathrm{z}}{ }^{\mathrm{mv}}(\omega)$ in Eqn (11) is proportional to $\omega^{3 / 2}$, which means that Rayleigh waves are generated more efficiently at higher frequencies (see also books [11, 12]). This explains why it is possible to neglect a bounce resonance of a car body and to use a 'body still' approximation in the quarter car model under consideration for calculation of generated ground vibrations.

Note that the Green's function used in Eqn (11) has been derived for a homogeneous elastic half space. Strictly speaking, it is not applicable to a layered soil, mainly because of a multi-modal and dispersive wave propagation in this case. In this situation one has to use a Green's function for a layered elastic half space (see e. g. $[13,14])$. This generally requires numerical calculations from the outset.

As our intention is to compare the results of the theoretical calculations with the experimental data obtained for the Leopard-1 MBT in the course of BVP [4], the selection of ground material constants had to be as consistent as possible with the ground parameters on the site of that experiments. The predominant soil type on the site of the experiments was sand, and thus the material parameters shown in Table 2, mass density $\rho$, Lame shear modulus $\mu$, and Poisson's ratio $\sigma=0.25$, represent typical values for this type of soil. Using the fact that for $\sigma=0.25$ the second Lamé constant $\lambda$ is equal to $\mu$, the values of elastic wave velocities in the ground have been calculated according to the well-known expressions $c_{l}=[(\lambda+2 \mu) / \rho]^{1 / 2}, c_{t}=(\mu / \rho)^{1 / 2}$, and $c_{R}=0.92 c_{t}$.

\subsection{Results of the calculations and discussion}


Theoretical ground vibration velocity spectra for the Leopard-1 MBT and for the Transportpanzer (Fuchs) APC at $11.8 \mathrm{~m}$ distance from the sensor and over time interval $T=$ 0.5 s calculated according to the Eqns (1), (2), (6), (7), (10) and (11) are shown in Figs 6 and 7 respectively. One can see that the calculated ground vibration velocity spectra largely repeat the main features of the corresponding ground force spectra (Figs 4 and 5). For the Leopard-1 MBT (see Fig. 6), these include major peaks at the track pitch frequency (23.1 $\mathrm{Hz}$ ) and its harmonics as well as smaller peaks at harmonics of the wheel-base frequency $(5.9 \mathrm{~Hz})$.

Like for the ground force spectra, there is a clear difference between the ground vibration spectrum generated by the APC wheeled vehicle and the one created by the tracked vehicle (MBT). For the Transportpanzer (Fuchs) APC (see Fig. 7), a single major peak is present within the range $0-200 \mathrm{~Hz}$ - at frequency associated with the tyre tread periodicity $(156 \mathrm{~Hz})$. The overall level of generated vibrations in this case is much lower than for the tank.

A comparison of the above-mentioned theoretical results with the experimental normalised power spectrum of ground vibrations generated by the Leopard-1 MBT at 11.8 $\mathrm{m}$ distance from the sensor [4] is given in Fig. 8. For convenience of comparison, the theoretical spectrum (Fig. 8,a) has been plotted for a square of ground vibration velocity, like the original experimental spectrum (see Fig. 8,b) that has been reproduced with kind permission from Fig. 8 of the paper [4]. Note that measurements in [4] have been processed using Hann window and determination of mean square spectral amplitudes, whereas in the present work no window and no mean square amplitudes have been simulated. Therefore, strictly speaking, one should not expect good agreement between the absolute levels of calculated and measured spectral peaks. 
It can be seen that the theoretical spectrum reproduces fairly well the experimental peaks associated with track periodicity (marked by circles in Fig. 8,b). However, there are some discrepancies. In particular, the magnitude corresponding to the fundamental frequency $(23.1 \mathrm{~Hz})$ is essentially lower in the experimental data. As it was mentioned in Reference [4], for tracked vehicles at low speeds the second multiple of the fundamental frequency was the strongest on passing the sensors (as it can be seen from Fig. 8,b), and for higher speeds, the fundamental frequency would produce the dominant response. A possible reason for this could be the effect of layered structure of the ground that has not been considered in the model. Also, the uncertainty in the ground attenuation coefficient assigned to the model could attribute to this and other observed differences between the theoretical and experimental data plots. The effect of ground attenuation is illustrated in Fig. 9 showing ground vibration spectra from Leopard-1 MBT for two values of ground loss factor: $\gamma=0.05$ and $\gamma=0.15$. And of course, the model does not reproduce the peaks in the experimental ground vibration spectrum associated with the engine frequency (19.65 $\mathrm{Hz}$ ) and its harmonics (marked by triangles in Fig. 8,b). Apparently, these peaks are due to acousto-seismic coupling [4, 9] of engine-radiated sound that has not been taken into account in this investigation.

Some of the observed differences between the obtained theoretical results and the experimental data could be attributed also to inaccuracy in modelling the track profile and to ignoring rotational motion of a vehicle body. Also, various additional generation mechanisms that have not been taken into account in this work could play a part. In addition to the already mentioned acousto-seismic coupling and ground stratification, these are the effects of engine vibrations due to rotating unbalance, spatial variations in ground elastic parameters and mass density in horizontal direction, ground topography, etc. Further 
research would be required to explore the effects of these missing mechanisms on ground vibration spectra generated by heavy military vehicles.

\section{Conclusions}

The results of this work show that analytical techniques based on the simplified quarter car vehicle model as well as on Green's function method are capable of producing ground vibration spectra generated by heavy military vehicles that reproduce basic properties of experimental spectra.

The established relationships between vehicle parameters and some characteristic features of generated ground vibration spectra can be considered as a starting point for much of additional research that is needed to arrive at the important goal of vehicle type identification. 


\section{References}

[1] Transportation noise: Reference book (ed. P. Nelson), Butterworths, London (1987).

[2] Noise and vibration from high-speed trains (ed. V.V. Krylov), Thomas Telford, London (2001).

[3] J. Altmann, S. Linev, A. Weiss, Acoustic-seismic detection and classification of military vehicles - Developing tools for disarmament and peace-keeping, Applied Acoustics, 63 (2002) 1085-1107.

[4] J. Altmann, Acoustic and seismic signals of heavy military vehicles for cooperative verification, Journal of Sound and Vibration, 273 (2004) 713-740.

[5] T.S. Anderson, M.L. Moran, S.A. Ketcham, J. Lacombe, Tracked vehicle simulations and seismic wavefield synthesis in seismic sensor systems, Computing in Science and Engineering, November/December, (2004) 22-28.

[6] S.A. Ketcham, M.L. Moran, J. Lacombe, R.J. Greenfeld, T.S. Anderson, Seismic source model for moving vehicles, IEEE Transactions on Geosciences and Remote Sensing, 43 (2005) 248-256. 
[7] V.V. Krylov, Generation of ground elastic waves by road vehicles, Journal of Computational Acoustics, 9 (2001) 919-933.

[8] V.V. Krylov, Generation of ground vibrations by accelerating and braking road vehicles, Acustica - Acta Acustica, 82 (1996) 642-649.

[9] J.M. Sabatier, H.E. Bass, L.N. Bolen, K. Attenborough, Acoustically induced seismic waves, Journal of the Acoustical Society of America, 80 (1986) 646-649.

[10] Jane’s Armour and Artillery 2008 - 2009 (ed. C.F. Foss), Jane’s Information Group Inc. (2008).

[11] W.M. Ewing, W.S. Jardetzky, F. Press, Elastic waves in layered media, McGrawHill, New York (1957).

[12] K.F. Graff, Wave motion in elastic solids, Clarendon Press, Oxford (1975).

[13] E. Kausel, J.M. Roesset, Stiffness matrices for layered soils, Bulletin of the Seismological Society of America, 71 (1981) 1743-1761.

[14] J.E. Luco, R.J. Apsel, On the Green’s functions for a layered half-space. Part I, Bulletin of the Seismological Society of America, 4 (1983) 909-929. 


\section{Figure captions}

Fig. 1. A quarter car vehicle model

Fig. 2. Leopard-1 MBT (a) and Transportpanzer (Fuchs) APC (b) (images are from Wikimedia Commons)

Fig. 3. Single-axle ground force spectrum calculated for the Leopard-1 MBT

Fig. 4. Multiple-axle ground force spectrum calculated for the Leopard-1 MBT

Fig. 5. Multiple-axle ground force spectrum calculated for the Transportpanzer (Fuchs) APC

Fig. 6. Ground vibration velocity spectrum at $11.8 \mathrm{~m}$ distance calculated for the Leopard-1 MBT

Fig. 7. Ground vibration velocity spectrum at $11.8 \mathrm{~m}$ distance calculated for the Transportpanzer (Fuchs) APC

Fig. 8. Comparison of the ground vibration spectrum at $11.8 \mathrm{~m}$ distance calculated for the Leopard-1 MBT - (a) with the corresponding experimental spectrum [4] - (b); the 
comparison is given for squares of vibration velocity as in the original Fig. 8 of paper [4], peaks associated with the main track frequency and its harmonics are marked with circles, triangles indicate harmonics of the engine frequency.

Fig. 9. Ground vibration velocity spectrum at $11.8 \mathrm{~m}$ distance calculated for the Leopard-1 MBT for two values of ground loss factor: $\gamma=0.05$ (solid curve) and $\gamma=0.15$ (dashed curve). 


\section{Table captions}

Table 1. Test vehicle parameters used in the calculations.

Table 2. Ground parameters used in the calculations; wave velocities shown have been calculated using the ground parameters given in the table. 
Figures

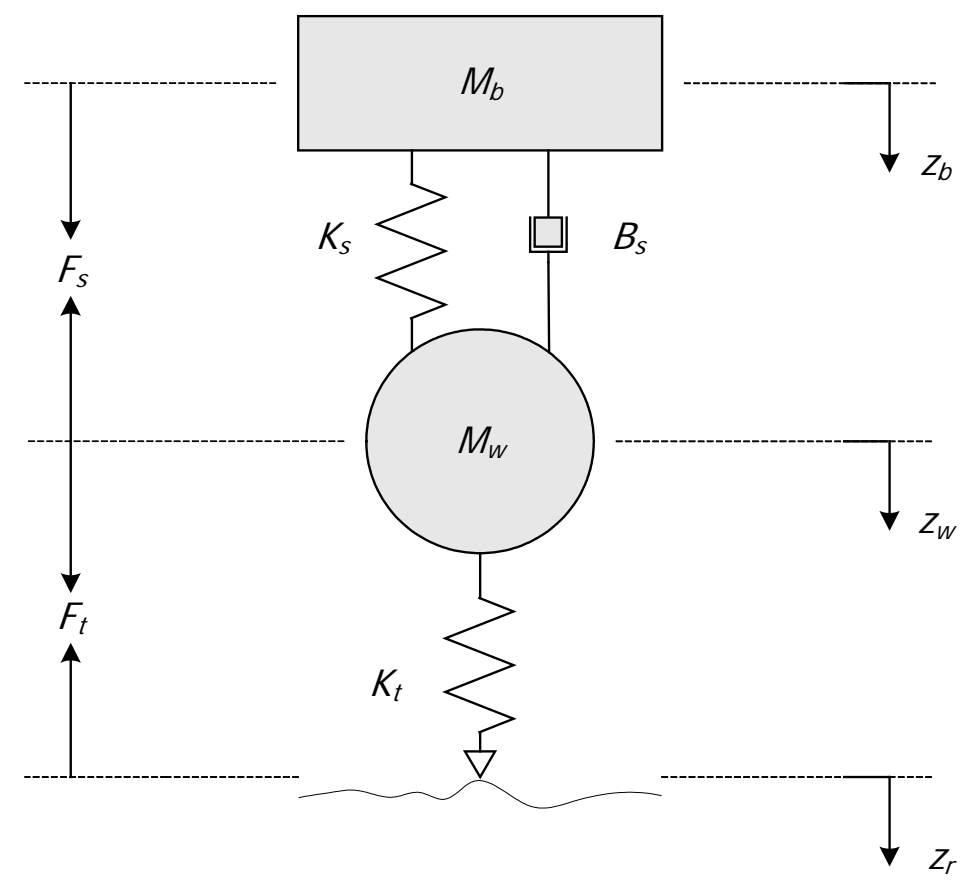

Fig. 1. A quarter car vehicle model 

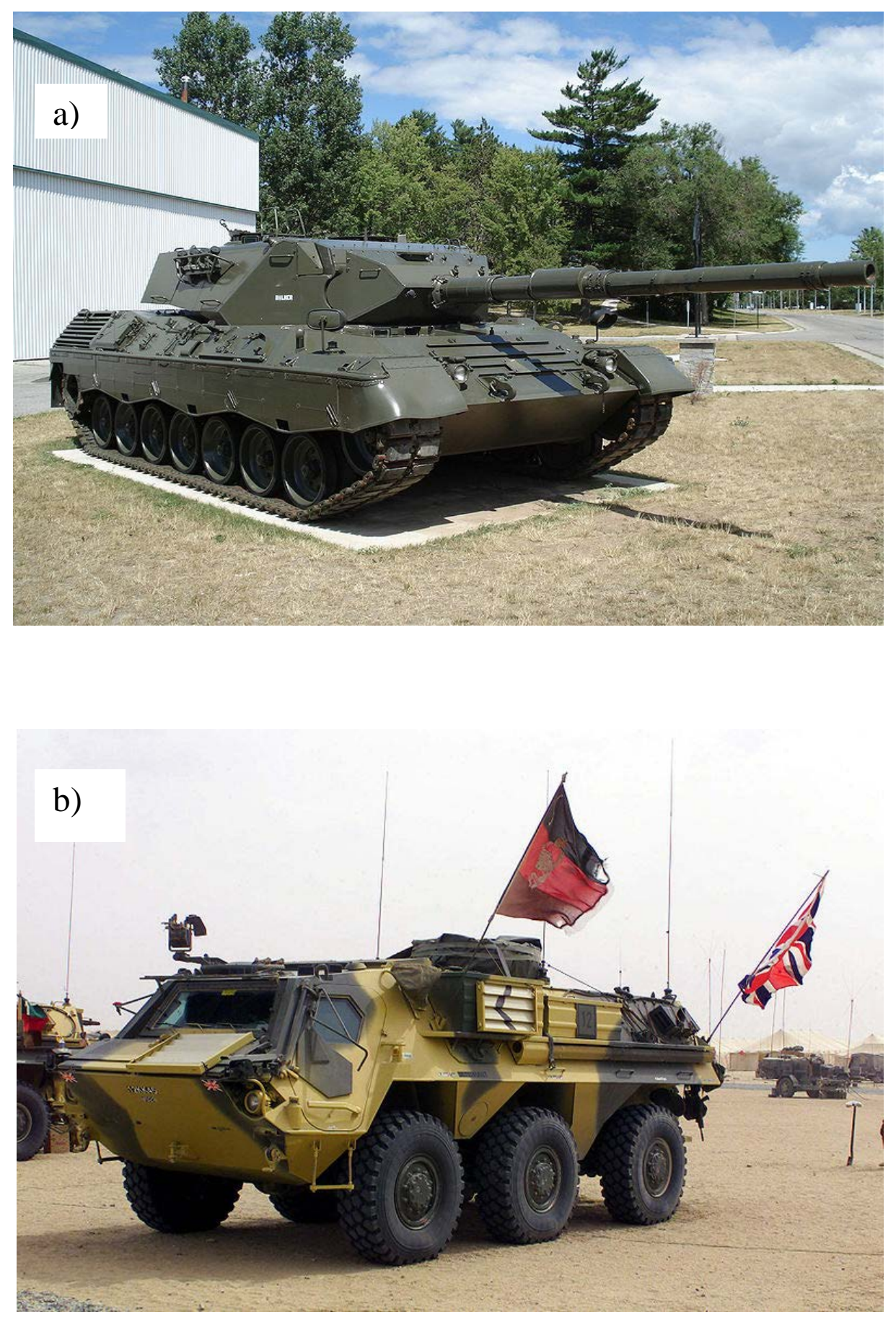

Fig. 2. Leopard-1 MBT (a) and Transportpanzer (Fuchs) APC (b) (images are from Wikimedia Commons) 


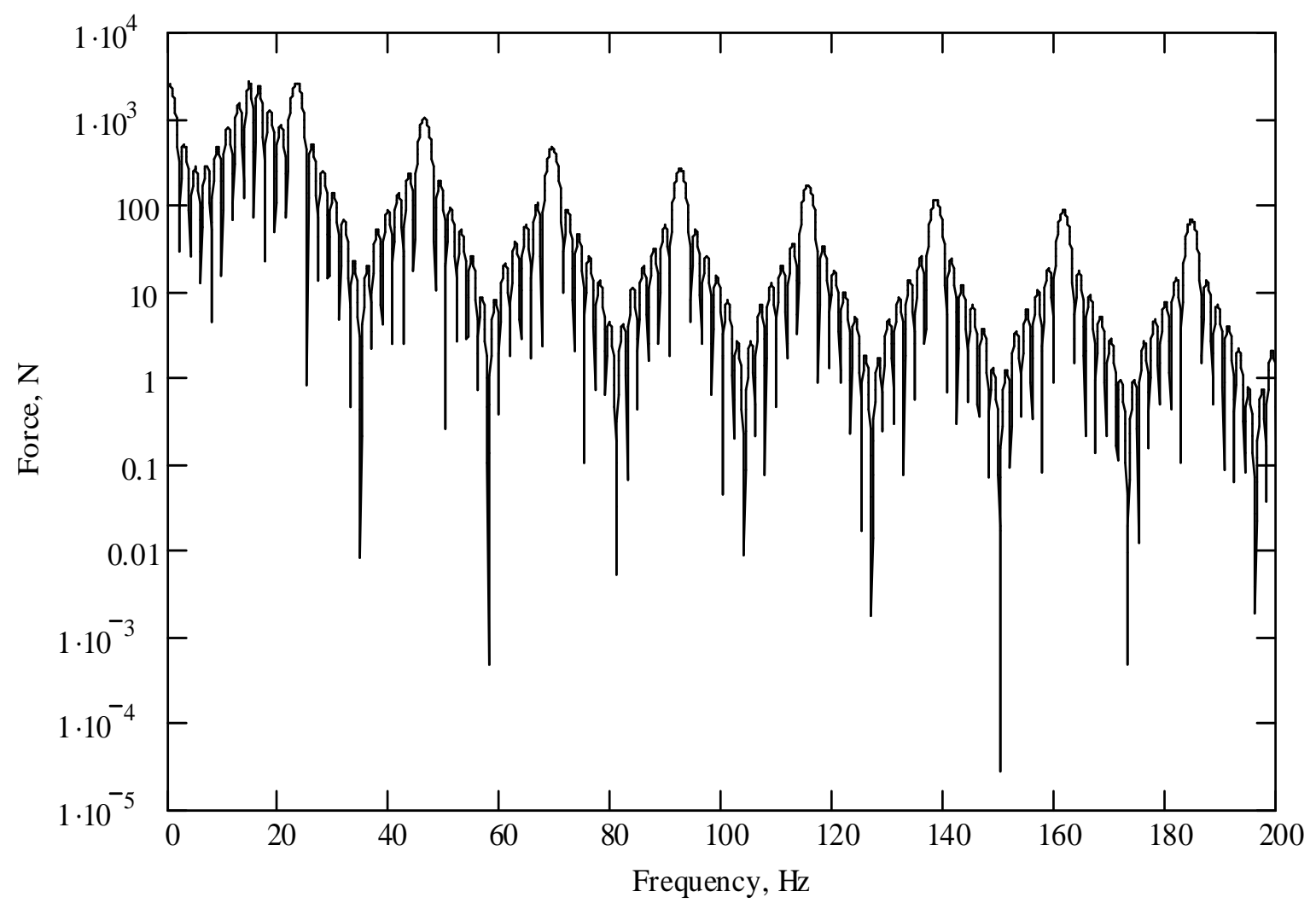

Fig. 3. Single-axle ground force spectrum calculated for the Leopard-1 MBT 


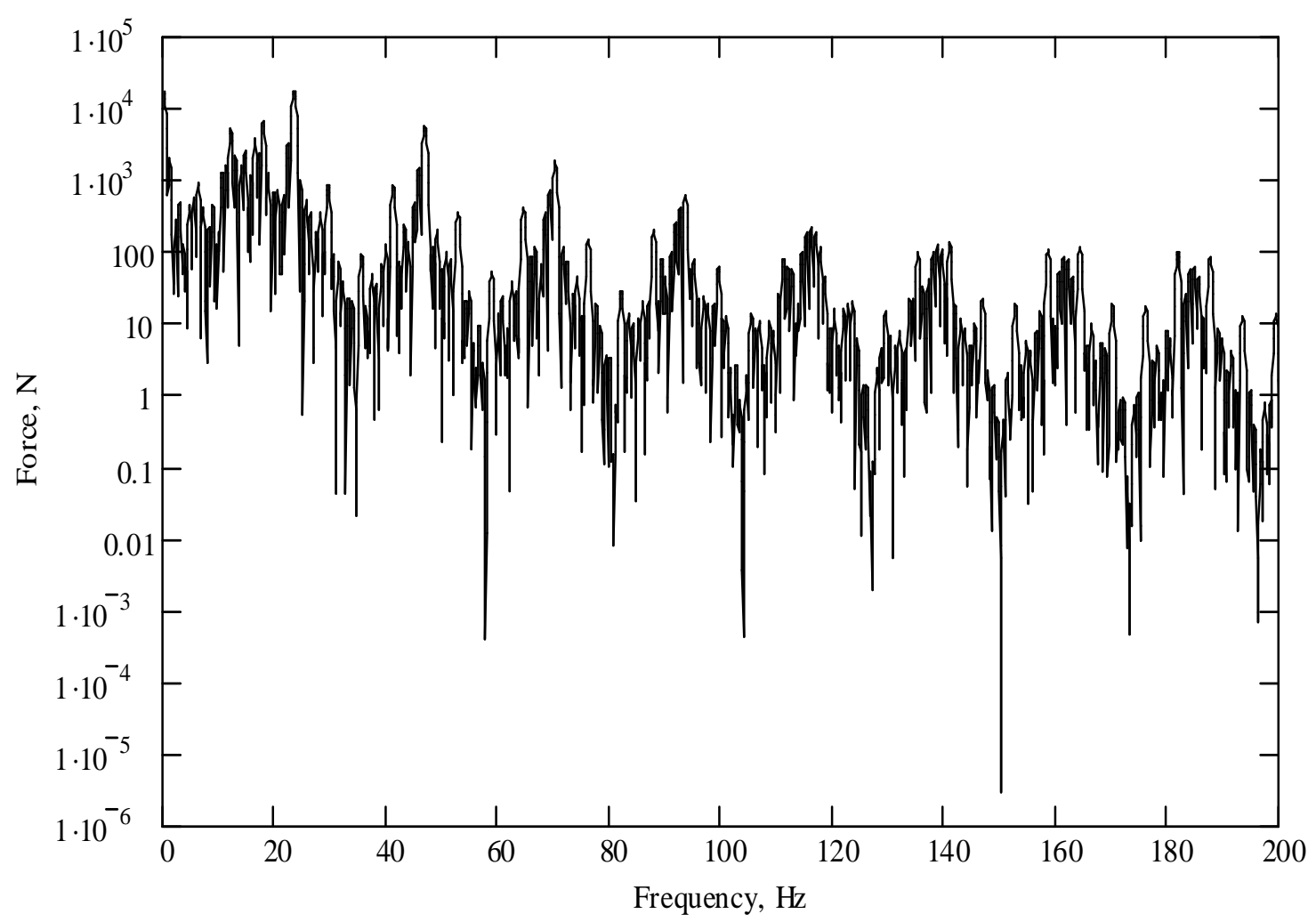

Fig. 4. Multiple-axle ground force spectrum calculated for the Leopard-1 MBT 


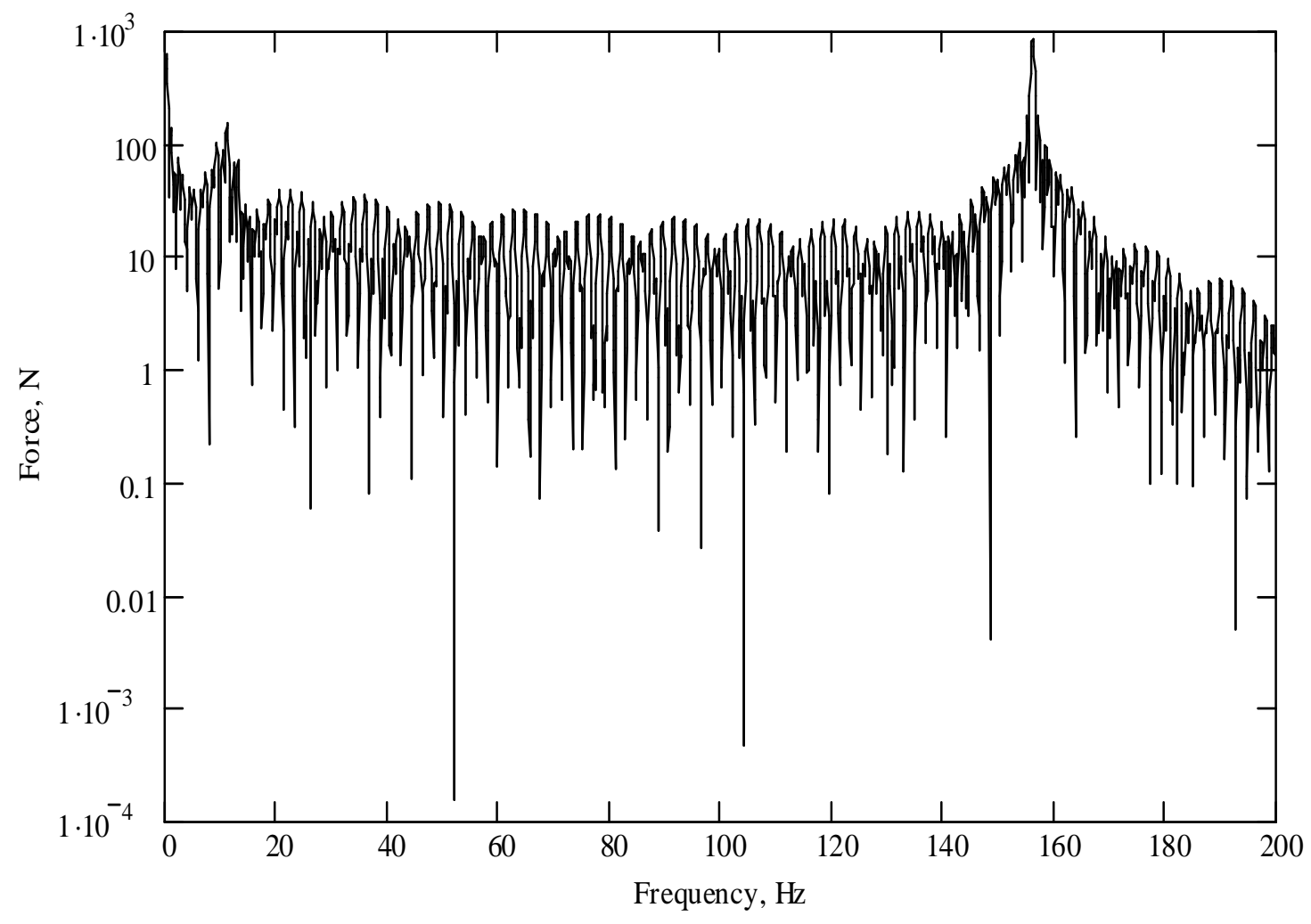

Fig. 5. Multiple-axle ground force spectrum calculated for the Transportpanzer (Fuchs) APC 


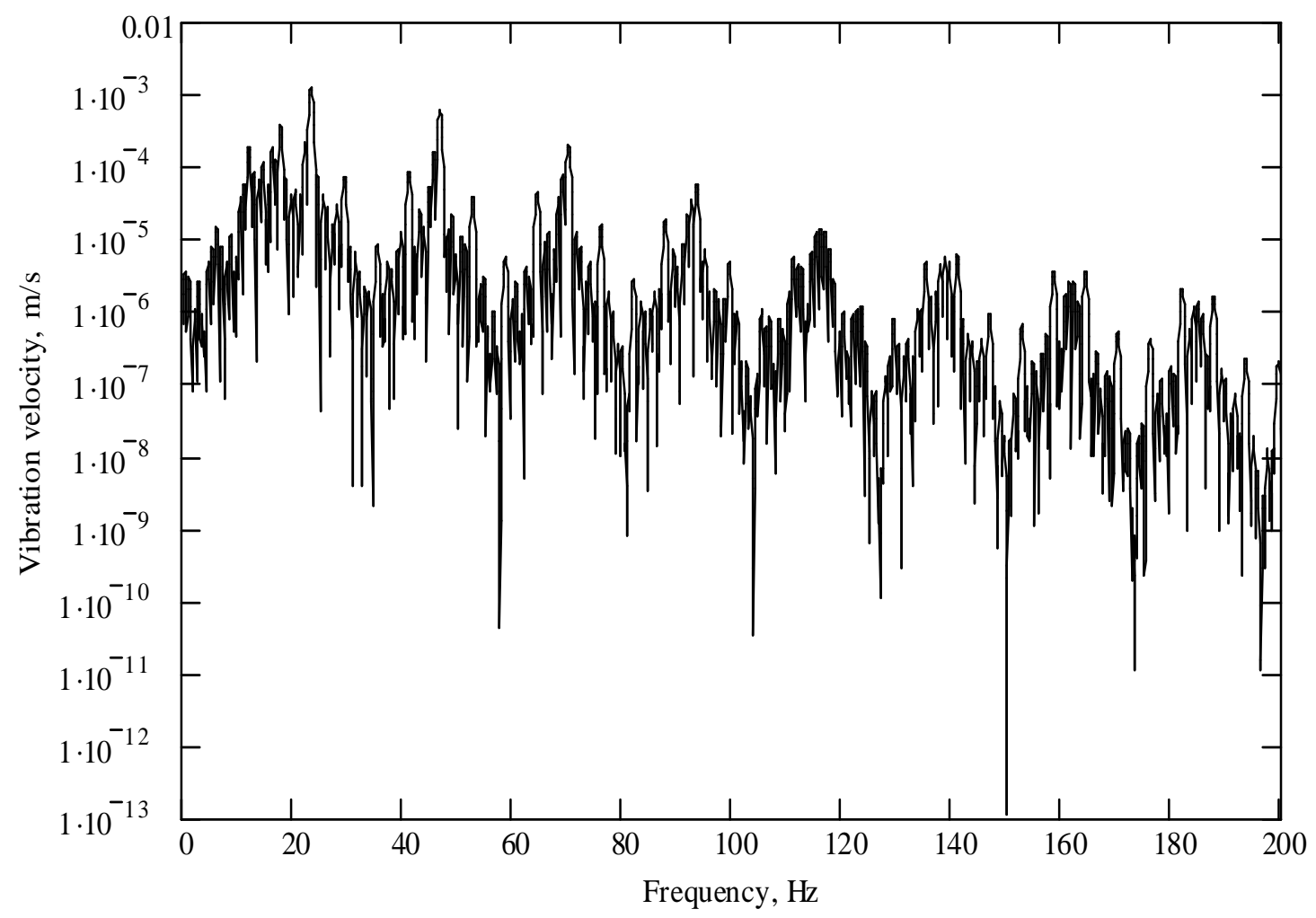

Fig. 6. Ground vibration velocity spectrum at $11.8 \mathrm{~m}$ distance calculated for the Leopard-1 MBT 


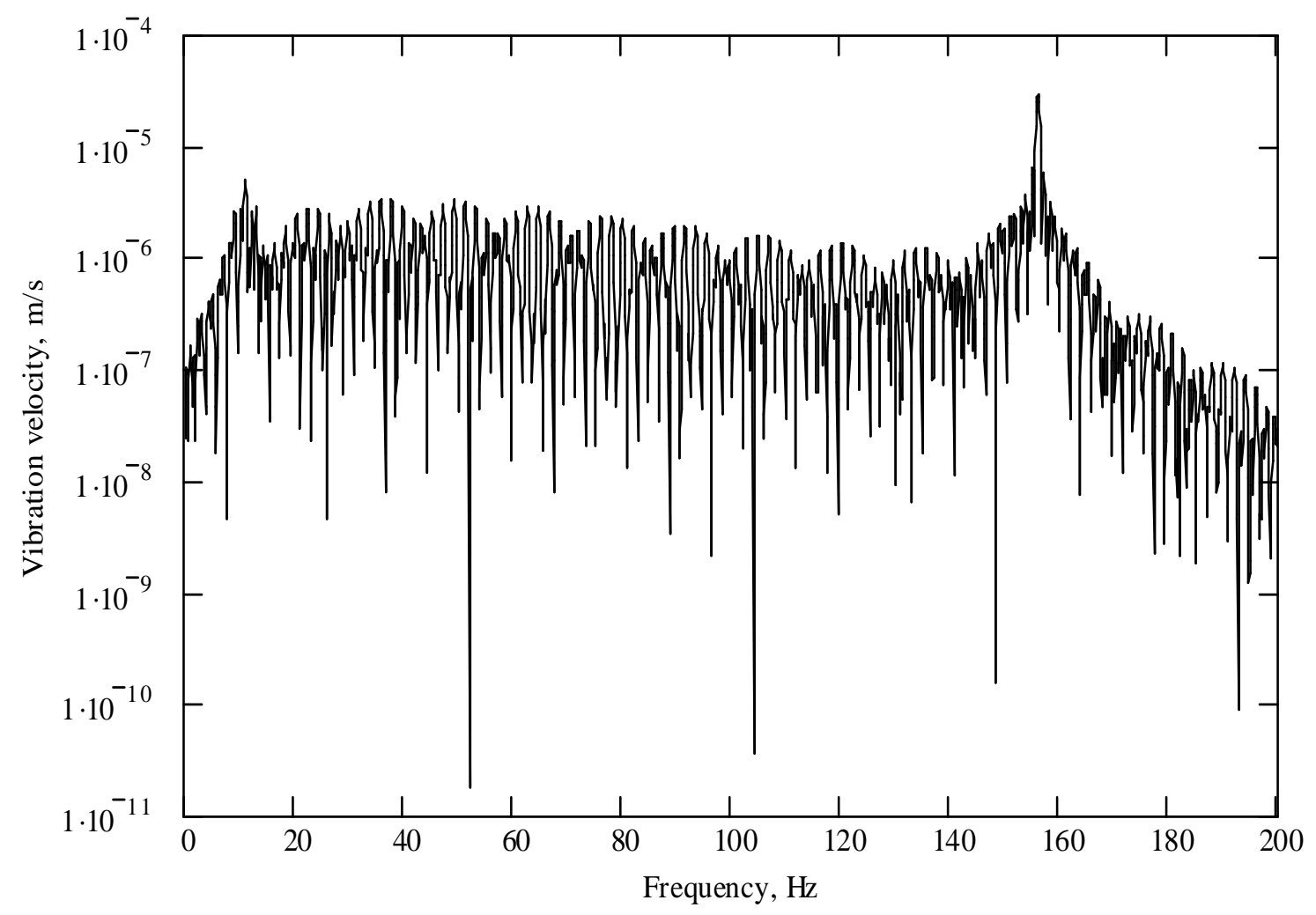

Fig. 7. Ground vibration velocity spectrum at $11.8 \mathrm{~m}$ distance calculated for the Transportpanzer (Fuchs) APC 

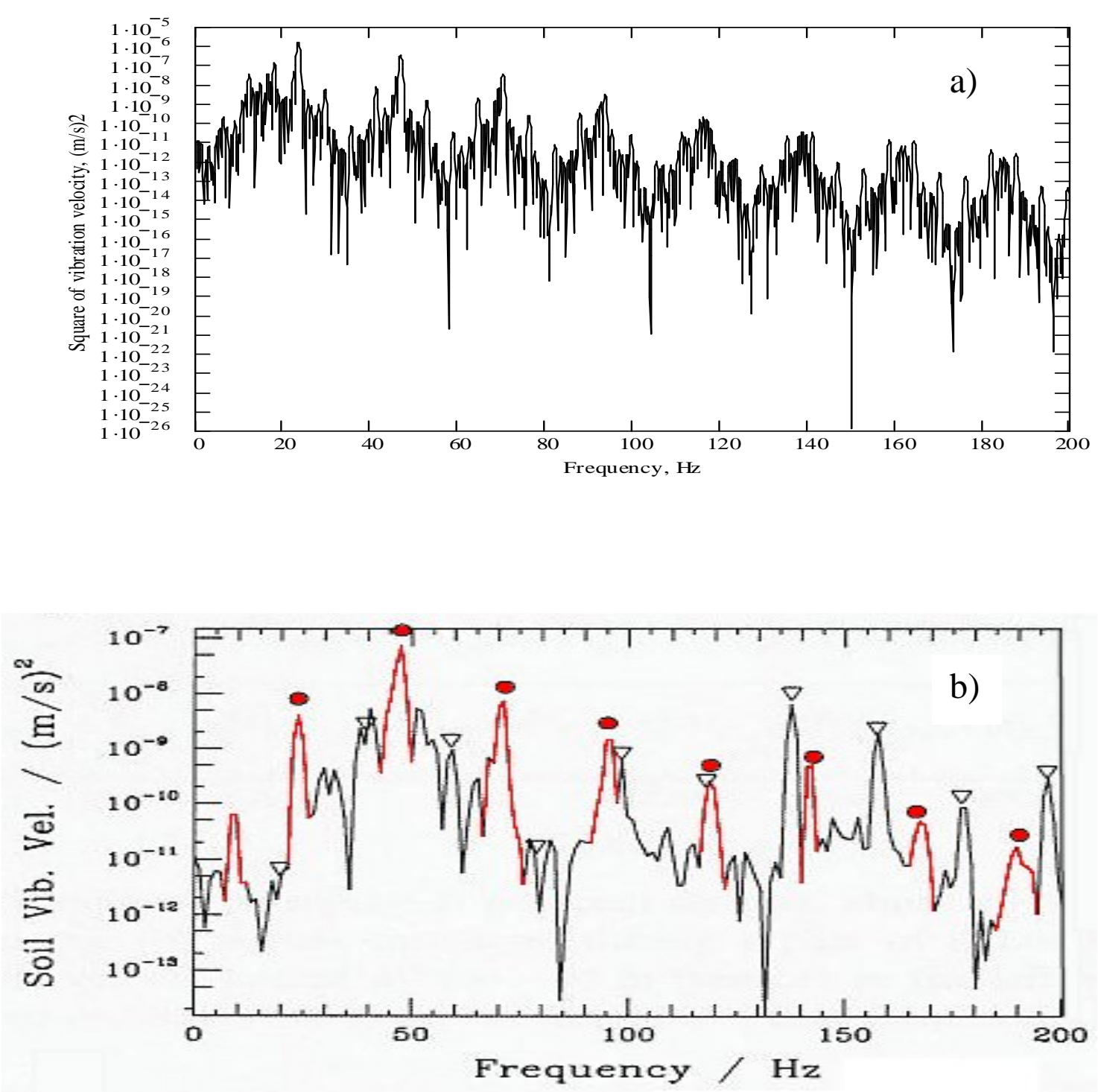

Fig. 8. Comparison of the ground vibration spectrum at $11.8 \mathrm{~m}$ distance calculated for the Leopard-1 MBT - (a) with the corresponding experimental spectrum [4] - (b); the comparison is given for squares of vibration velocity as in the original Fig. 8 of paper [4], peaks associated with the main track frequency and its harmonics are marked with circles, triangles indicate harmonics of the engine frequency. 


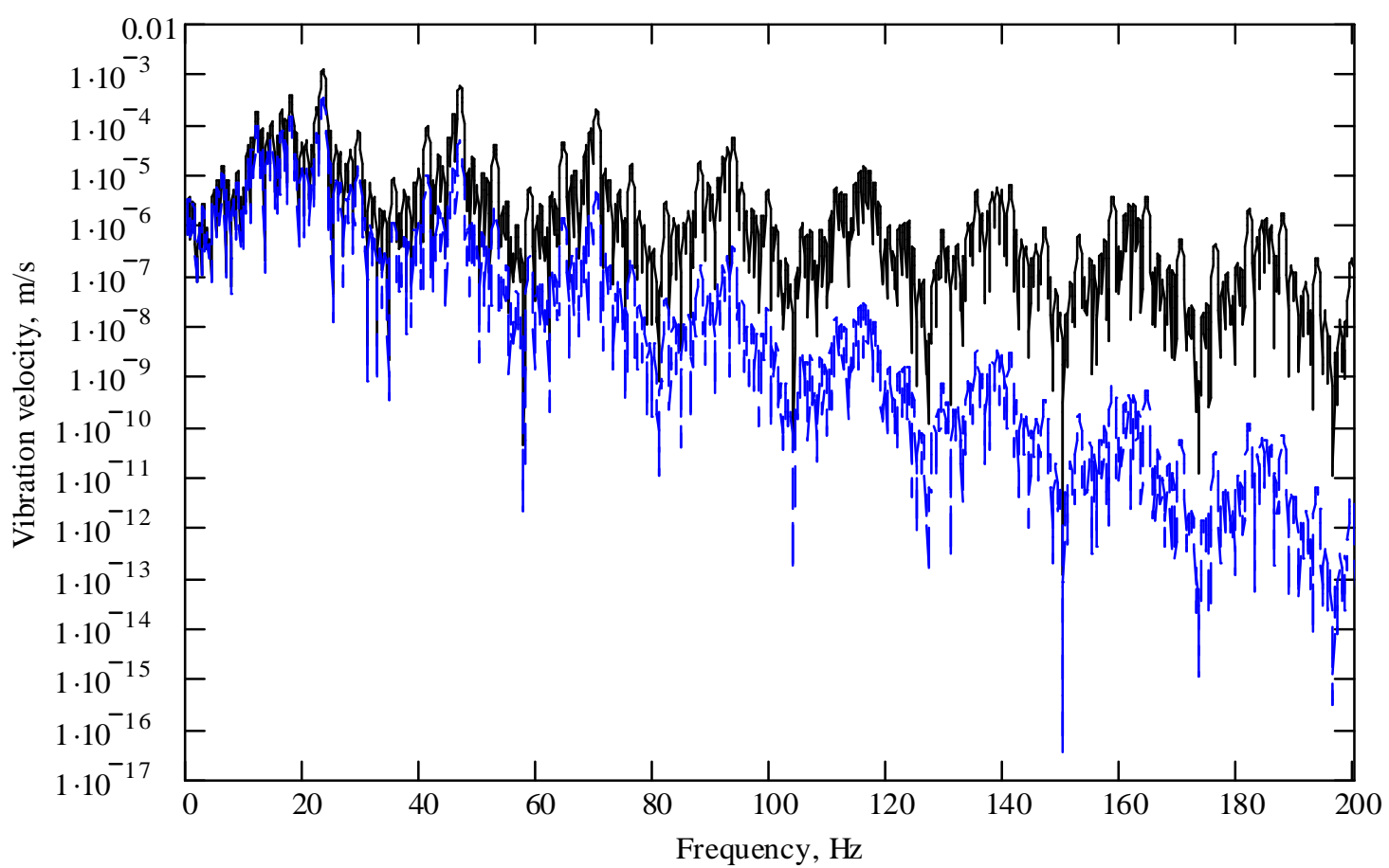

Fig. 9. Ground vibration velocity spectrum at $11.8 \mathrm{~m}$ distance calculated for the Leopard-1 MBT for two values of ground loss factor: $\gamma=0.05$ (solid curve) and $\gamma=0.15$ (dashed curve). 


\section{Tables}

\begin{tabular}{|l|l|l|l|}
\hline \multicolumn{1}{|c|}{ Quarter car model parameters } & \multicolumn{1}{|c|}{$\begin{array}{c}\text { Symbol, } \\
\text { Unit }\end{array}$} & \multicolumn{1}{c|}{$\begin{array}{c}\text { Leopard-1 } \\
\text { MBT }\end{array}$} & \multicolumn{1}{c|}{ Transportpanzer } \\
(Fuchs) APC
\end{tabular}

Table 1. Test vehicle parameters used in the calculations. 


\begin{tabular}{|l|l|l|}
\hline \multicolumn{1}{|c|}{ Ground parameters } & Symbol, Unit & \multicolumn{1}{c|}{ Value } \\
\hline Mass density & $\rho, \mathrm{kg} \mathrm{m}^{-3}$ & 1800 \\
\hline Shear modulus & $\mu, \mathrm{Nm}^{-2}$ & $4 \times 10^{7}$ \\
\hline Loss factor & $\gamma$ & 0.05 \\
\hline Poisson's ratio & $\sigma$ & 0.25 \\
\hline Longitudinal wave velocity & $\mathrm{c}_{\mathrm{l}}, \mathrm{ms}^{-1}$ & 258.2 \\
\hline Shear wave velocity & $\mathrm{c}_{\mathrm{t}}, \mathrm{ms}^{-1}$ & 149.1 \\
\hline Rayleigh wave velocity & $\mathrm{c}_{\mathrm{R}}, \mathrm{ms}^{-1}$ & 137.2 \\
\hline
\end{tabular}

Table 2. Ground parameters used in the calculations; wave velocities shown have been calculated using the ground parameters given in the table. 